\title{
Prospects for advancing nuclear cardiology by means of new detector designs
}

\author{
Kenneth J. Nichols, PhD, ${ }^{\text {a }}$ Andrew Van Tosh, MD, ${ }^{\mathrm{b}}$ \\ and Christopher J. Palestro, $M^{a}$
}

In the March/April issue of the Journal, there is a fascinating article by Slomka et $\mathrm{al}^{1}$ reporting advances in SPECT detector design and image reconstruction techniques. In this essay we shall outline briefly the physical principles that have made these innovations possible and explore ways in which diagnosing cardiac disease may be advanced with this new technology.

First, it is useful to understand how it has become possible to improve Nuclear Cardiology image quality. Usually, for any given technology, such as Anger camera tomograms reconstructed by filtered backprojection, improving one property of an image causes degradation of other image properties; for instance, improving signal-to-noise ratio by applying a stronger spatial filter decreases image contrast and spatial resolution. ${ }^{2}$ In order to achieve a genuine improvement in image quality it is necessary to replace an older technology with a newer one. One way to accomplish this is to replace filtered backprojection reconstruction approaches with more sophisticated iterative reconstruction algorithms; another approach is to replace the Anger cameras with superior data collecting devices.

Ultimately, the reliability of scintigrams is inseparably connected to the amount of information associated with each detected gamma ray. Anger cameras consist of a single large $\mathrm{NaI}(\mathrm{Tl})$ crystal with a bank of many photomultiplier tubes and a collimator. Some of the recent improvements in image quality have come about by replacing the $\mathrm{NaI}(\mathrm{Tl})$ crystal with a solid state device, such as CZT and CSI(Tl) crystals, which provide considerably more information for each detected gamma ray. For instance, every time a $140 \mathrm{keV}$ gamma ray scintillates in a $\mathrm{NaI}(\mathrm{Tl})$ crystal, it produces 5,600 light

\footnotetext{
From the Division of Nuclear Medicine and Molecular Imaging, North Shore-Long Island Jewish Health System, New Hyde Park, NY; Research Department, ${ }^{\text {b }}$ St. Francis Hospital, Roslyn, NY.

Reprint requests: Kenneth J. Nichols, PhD, Division of Nuclear Medicine and Molecular Imaging, North Shore-Long Island Jewish Health System, 270-05 76th Avenue, New Hyde Park, NY 11040; knichols@lij.edu.

J Nucl Cardiol 2009;16:691-6.

$1071-3581 / \$ 34.00$

Copyright $(2009$ by the American Society of Nuclear Cardiology. doi:10.1007/s12350-009-9123-8
}

photons, which are converted to 700 photoelectrons, which then must be amplified in a photomultiplier tube to produce an electronic signal suitable for information processing. ${ }^{3}$ The greatest factor contributing to Anger camera dead time arises from the need to compute the location of the scintillation within a large $\mathrm{NaI}(\mathrm{Tl})$ crystal from the pattern of currents flowing through many photomultiplier tubes. In contrast, if a $140 \mathrm{keV}$ gamma ray encounters a CZT detector, its energy is converted directly to 30,000 electron-hole pairs, so that much more information is generated per detection, and the signal is immediately available for information processing. This translates into considerably better energy resolution, the implications of which are described below.

The small size of solid state detectors (only a few millimeters thick) has made possible innovative detector designs, including few or no moving parts (other than allowing detectors to be positioned as closely to the patient as possible), that are arranged to provide threedimensional image formation. Imaging with some of the newer detector designs is similar to PET imaging, which also uses stationary detectors and which acquires data three-dimensionally. A stationary detector system has the practical advantage of having fewer breakdowns and reduced detector down time.

Conventional filtered backprojection applied to multiple planar projections processes information from only one plane at a time. Filtered backprojection reconstructs information in a plane as if there is no relation between that information and information in contiguous planes, whereas the information contained in contiguous transaxial planes is highly correlated. Newer solid state detector designs lend themselves well to true threedimensional acquisition of SPECT data. The formation of tomograms by combining information from many different small detectors is implemented through the use of newer, sophisticated, maximum likelihood expectation maximization (MLEM) algorithms, ${ }^{4}$ and ordered subset expectation maximization (OSEM) algorithms. ${ }^{5}$ MLEM and OSEM algorithms not only generate the tomogram, but also make it possible to correct for estimated amounts of scattered radiation, by using information in multiple energy windows. ${ }^{6,7}$ At the same time, these algorithms enable the incorporation of detailed knowledge about the 
manner in which detector response varies with the distance away from each detector, ${ }^{8}$ and attenuation correction information, such as from a CT scan.

Anger cameras typically have count sensitivities in clinical studies of 0.5-0.7 kcps and energy resolution of 9-10\%, with tomographic spatial resolution on the order of 9-11 mm. In contrast, CZT SPECT cameras have sensitivities in clinical studies of 2.2-4.7 kcps, energy resolution of $5.7 \%$, and tomographic spatial resolution ranging from 4.3 to $4.9 \mathrm{~mm} .{ }^{9,10}$ This amounts to simultaneously acquiring 3-5 times more gamma rays, or even 10 times more counts, ${ }^{11}$ with 1.65 times more precisely defined gamma ray energy, and with 1.7-2.5 times better spatial resolution than the Anger camera. ${ }^{9}$ What are the likely advantages of these improvements in SPECT image characteristics for diagnosing cardiac disease?

\section{IMPROVED ENERGY RESOLUTION}

Overall image quality is related to energy resolution. There always is a limit to the precision with which the energy of detected gamma rays can be measured, regardless of the detector used. Consequently, some gamma rays reported as having $140 \mathrm{keV}$ of energy are actually lower or higher energy scattered gamma rays that did not actually originate from the direction computed by the detector's spatial logic circuitry. Rather, these are scattered gamma rays emitted at other locations within the patient's body. Including them in reconstructed tomograms causes the tomograms to appear to have concentrations of radionuclides in areas where there are no isotopes. Yet, it is necessary to set a sufficiently wide energy window in deciding which detected gamma ray events to process, because even true $140 \mathrm{keV}$ primary energy gamma rays can be reported to have energies lower or higher than $140 \mathrm{keV}$. Failing to set a sufficiently wide energy window will result in needlessly discarding true primary gamma rays. Usually the energy window is set sufficiently wide to include 95\% of likely computed energies that the detector is expected to report as a result of detecting primary gamma rays. The advantage of using technology with $5-6 \%$ energy resolution, as opposed to 9-10\% energy resolution, is that the reconstructed tomogram will contain fewer scattered gamma rays. As a result, tomograms acquired with better energy resolution will have higher image contrast and an improved ability to discriminate areas with isotope uptake versus those without tracer uptake. Areas of relatively less myocardial uptake contiguous to areas of normal perfusion should be seen more easily because of higher image contrast, and be more accurately localized.

Improved energy resolution should facilitate the implementation of dual isotope imaging protocols. One example is rest Tl-201 collected simultaneously with stress Tc-99m-sestamibi count distributions, both acquired under resting conditions. While there have been attempts to implement this protocol using Anger camera technology, ${ }^{12}$ it has been challenging to accomplish due to considerable overlap of Tc-99m gamma rays scattered down into the Tl-201 69-81 keV energy window. Solid state detectors with improved energy resolution and higher sensitivity should facilitate acquisition of Tl-201 images with a larger number of $167 \mathrm{keV}$ Tl-201 gamma rays, and improve the likelihood of separating Tc-99m images from Tl-201 images. It would be advantageous to be able to image the resting perfusion distribution with Tl-201, with its favorable resting tracer kinetics, ${ }^{13}$ registered to the same locations as the sestamibi stress distribution. This would obviate possible misregistration problems inherent in acquiring data during two separate imaging sessions, as well as reduce the possible complication of left ventricular (LV) shape differences for stress versus rest studies that can occur in transient ischemic dilatation following stress testing. ${ }^{14}$ Another potential application is simultaneous performance of Tl-201 and I-123-MIBG imaging, which could aid in assessing adrenergic activity and myocardial perfusion simultaneously in patients with CHF. ${ }^{15}$

\section{INCREASED COUNT SENSITIVITY}

Recently, newer reconstruction algorithms, such as the MLEM and OSEM approaches mentioned above, have been applied to Nuclear Cardiology applications that enable Anger camera tomograms in half the time with the same image quality as had previously been achieved with conventional filtered backprojection. ${ }^{16-19}$ Yet, in assessing the quality of those tomograms, observers find that in a small percent of scans the problem continues to be inadequate counts, producing images that readers judge as being of poor quality. This can happen if patients are unusually obese, or if myocardial uptake is unusually reduced. In triple vessel disease, all coronary arteries are partially blocked, so that radiotracer uptake is reduced in a balanced fashion that is difficult to detect. ${ }^{20,21}$ Coronary vasospasm and previous ischemia also can result in reduced tracer uptake. ${ }^{22}$ Although increasing the amount of radioactivity injected can compensate for this, it is not desirable to do so.

In order to use an Anger camera it must be equipped with a collimator (usually parallel-hole), which enables defining the direction of travel of the gamma rays at the expense of low sensitivity, admitting only 1 out of every 10,000 gamma rays emitted by the patient. ${ }^{3}$ Newer solid state detector designs incorporate collimators too, but 
their more efficient energy conversion processes provide higher count rates. The prospect of acquiring 3-5 times more counts in the same amount of time should translate into improved image quality.

Initial reports indicate that myocardial perfusion image quality achieved with the newer solid state cameras in 5-6 minutes is comparable or better than what is obtained with a conventional Anger camera in 14-15 minutes. ${ }^{23,24}$ Since newer detectors acquire 3-5 times more data than conventional SPECT, acquisition times of 2 minutes, or less, might be feasible. This should reduce the frequency of patient motion artifacts and improve laboratory efficiency. In clinical trials involving large sample sizes, the newer detectors provide images that are at least comparable to those obtained with conventional SPECT cameras, ${ }^{11,25,26}$ so the newer designs could well provide both improved laboratory efficiency and improved diagnostic image quality. The possibility of acquiring clinically useful SPECT data in only 2 minutes could permit protocols in which the heart is imaged during stress, to capture abnormal functional as well as abnormal perfusion information, to be compared to resting function and perfusion in a separate acquisition.

In the same way that improvements in reconstruction algorithms of data collected by conventional SPECT cameras have made it feasible to collect gated SPECT myocardial perfusion data at frame rates of 24 cine frames/R-R interval, ${ }^{27}$ thereby likely resulting in more precise diastolic and systolic functional parameters, higher cinematic frame rates should be feasible with the newer solid-state detectors owing to their greater count sensitivity. The prospect of routinely acquiring more reliable peak ejection rate and peak filling rate parametric data through higher sampling rates for LV volume versus time curves should add a further dimension to the quantification of cardiac disease.

Another potential benefit of increased sensitivity is decreased radiation dose to the patient. There is particular concern regarding limiting radiation dose in conjunction with 64-slice CT scanning in pediatric studies, ${ }^{28}$ and in studies involving younger women. ${ }^{29}$ This is especially worth noting for laboratories that intend to perform cardiac SPECT studies in conjunction with 64-slide CT scans, for which surface doses can be high. ${ }^{30}$ So, while interest has been focused on reducing scan times using SPECT detector technology, an additional benefit could be the ability to tailor protocols to deliver a substantially smaller amount of injected activity to younger patients and maintain the same amount of time (10-14 minutes) to perform a SPECT scan.

Another means by which the newer detectors could impact laboratory operations is to make it feasible to acquire the same number of cardiac counts for each patient, thereby providing a measure of consistency from one patient to the next. Rotating Anger cameras can be used this way now, by first ascertaining count rates using an initial 1-minute scan and adjusting the acquisition time per projection, ${ }^{31,32}$ but this procedure is not widely implemented. Because the newer devices acquire data three-dimensionally, acquisition computers could easily be set to collect a predetermined amount of counts without first acquiring a 1-minute calibration study. The advantageous imaging properties of the newer detector designs should provide considerably more flexibility in designing diverse imaging protocols, and tailoring them to particular patient groups.

Along with the accumulation of more counts and higher count rates should come the ability to determine absolute uptake in units of $\mathrm{kBq} / \mu \mathrm{g} .{ }^{33,34}$ This always has been desirable, but has not been implemented for SPECT, in part due to inadequate count statistics. In positron imaging, the PET camera is calibrated using a well counter, and the syringe used to deliver the tracer is measured before and after injections to enable absolute quantitation. There will be no reason not to do this for the newer SPECT cameras.

First pass transit studies, a staple of Nuclear Cardiology in the 1970s, could become relevant again because of the potential for measuring coronary flow reserve (CFR). Rb-82 PET imaging has the capability to provide absolute measurement of myocardial blood flow (MBF), ${ }^{35,36}$ and CFR, ${ }^{37}$ routinely. Now it may be possible for SPECT to do that too. CFR measurement requires reliable count rate data for both the bolus injection phase and for the subsequent equilibrium blood pool phases of tracer injection, during stress and at rest. Many conventional Anger cameras are incapable of providing sufficiently high quality data because their sensitivity decreases drastically when presented with too high a count rate during the injection phase. However, solid state detectors have much shorter dead time, so that acquiring such data may well be feasible.

\section{IMPROVED SPATIAL RESOLUTION}

It can be quite difficult to distinguish between myocardial perfusion defects caused by disease of the right coronary from those caused by disease of the distal left anterior descending artery, not only for SPECT but also for PET, ${ }^{38}$ and to determine if one or more arteries are involved. The ability to resolve smaller structures with the new technologies should provide higher sensitivity to detect smaller, more subtle lesions, and to distinguish multi-vessel from single vessel disease. Being able to see suggestions of papillary muscles and atria should provide a higher degree of confidence in 
localizing perfusion and functional abnormalities. The more anatomic clues that are available from the SPECT data, the higher the degree of confidence in matching SPECT to CT images to verify, or correct, the registration between SPECT and CT scans.

It is possible that more accurate estimates of $\mathrm{LV}$ mass may become available from SPECT data. Until now, tests for detecting unusually thick myocardium in conventionally acquired gated SPECT data have found no convincing evidence that patients' myocardium ever exceeds the partial volume limit, ${ }^{39}$ even in individuals with LVH. ${ }^{40}$ The normal myocardial thickness is on the order of $1 \mathrm{~cm}$ thick at end diastole, which is about twice the spatial resolution of the newer cameras. So, it may become possible to detect instances in which the count profile across the myocardium is not simply the detector's line spread function, but which would actually constitute evidence of unusually thick myocardium. Clearly, echocardiography is excellent for quantifying myocardial mass, and it is unlikely SPECT would be performed solely for this purpose. Rather, as LVH is associated with potentially serious health implications, an incidental finding of unusually thick myocardium seen on gated SPECT studies would trigger further investigations.

Depicting at least some of the right ventricular (RV) myocardium a greater percent of the time for a greater percent of the RV also may become feasible. This could constitute a new realm for diagnosis of cardiac disease by SPECT. Currently a finding of reduced apical perfusion is not sufficient to identify a problem with the LV or RV. The ability to recognize RV infarction, specifically, should promote a better understanding of the disease state of an individual. Currently gated blood pool SPECT imaging is the only Nuclear Cardiology method available for recognizing regional RV motion and phase abnormalities. ${ }^{41,42}$ If similar information can be obtained from gated myocardial perfusion studies, it could shed light on a number of cardiac and pulmonary disease processes associated with RV disease, potentially providing better patient management.

Improved spatial resolution will likely reduce the tendency of functional quantitative algorithms to underestimate smaller ventricular volumes and overestimate high ejection fraction (EF). High EFs are not given as much credence when derived from myocardial perfusion SPECT imaging, due to the reliance of the calculations on quantified volumes. When myocardial walls in smaller hearts contract, counts from one wall spill over into the LV cavity at systole, causing artifactually increased contraction. Improved spatial resolution likely will reduce this phenomenon and, in conjunction with the acquisition of more counts, should provide more accurate EFs.

\section{CHALLENGES}

Verification that imaging with newer solid-state detectors provides image quality equal to or superior to that obtained with conventional Anger camera SPECT is essential, of course, but validations of perfusion and function parameters versus independent reference standards, such as CT, cardiac MRI, or x-ray contrast arteriography, are also needed. Some reports have found that LV EF and volume values are systematically lower when conventional Anger cameras use MLEM and OSEM reconstruction algorithms. ${ }^{18,19}$ Newer solid state detectors form reconstructions using OSEM algorithms, and consequently, verification of LV myocardial perfusion and function quantification versus independent reference standards is important. Data need to be collected in actual clinical settings to obtain a realistic idea of quantifiable improvements in accuracy of perfusion defect size, location, and severity, as well as functional parameters. This is particularly relevant considering that imaging with solid-state detectors is likely to produce images of better quality than Anger camera SPECT, so only comparing against conventional SPECT will not provide a complete picture of improvements in accuracy. Considering that diagnosis of cardiac disease is already highly successful with conventional Anger cameras, and that the newer detectors undoubtedly will be more expensive than conventional systems, it will be necessary to prove definitively that they provide significantly better sensitivity, specificity, and accuracy in order for these new systems to gain acceptance.

While improvement in spatial resolution is highly desirable, the ability to image smaller structures may well require adjustments to existing Nuclear Cardiology algorithms. Gated SPECT data sets of improved spatial resolution may present challenges to assumptions about relationships between percent wall thickening and systolic count increases, ${ }^{43}$ because the LV myocardium at end diastole now may have a thickness of about twice the spatial resolution of some of the new devices. The commercially available Nuclear Cardiology algorithms that compute LV volumes and EFs incorporate assumptions about wall thickening by way of systolic count increases, ${ }^{44,45}$ so it may be necessary to make appropriate adjustments to these algorithms or even to develop entirely new algorithms.

Shorter acquisition times made possible by the newer detector designs should reduce the frequency of motion artifacts, but it will not eliminate them. Detectors that acquire information three-dimensionally still will be blurred by "upward creep" of the heart as the diaphragm relaxes. ${ }^{46}$ The washout of Tl-201, which can cause the appearance of the abrupt jump of projection data acquired by two-detector data, will not appear, yet 
will still alter count rate information regionally during the course of data acquisition. These types of artifacts possibly could be post-corrected if data are collected in gated list mode, but new, as yet unanticipated, artifacts may appear with this new technology. It will be necessary to be on the alert for new kinds of artifacts.

\section{SUMMARY}

In summary, the newer detector designs offer the possibility of improved Nuclear Cardiology image quality and suggest a wide range of new, innovative imaging protocols. They may enable obtaining more accurate and more varied quantified perfusion and functional parameters.

\section{References}

1. Slomka PJ, Patton JA, Berman DS, Germano G. Advances in technical aspects of myocardial perfusion SPECT imaging. J Nucl Cardiol 2009;16:255-76.

2. Cullom SJ. Principles of cardiac SPECT imaging. In: DePuey EG, Berman DS, Garcia EV, editors. Cardiac SPECT. 2nd ed. Philadelphia: Lippincott, Williams \& Wilkins; 2001. p. 3-16.

3. Cherry SR, Sorensen JA, Phelps ME. Physics in nuclear medicine. 3rd ed. Philadelphia: WB Saunders; 2003. p. 211-26.

4. Shepp LA, Vardi Y. Maximum likelihood reconstruction for emission tomography. IEEE Trans Med Imaging 1982;1:113-22.

5. Hudson HM, Larkin RS. Accelerated image reconstruction using ordered subsets of projection data. IEEE Trans Med Imaging 1994;13:601-9.

6. Bowsher JE, Floyd CE. Treatment of Compton scattering in maximum likelihood, expectation-maximization reconstructions of SPECT images. J Nucl Med 1991;32:1285-91.

7. Xiao J, de Wit TC, Staelen SG, Beekman FJ. Evaluation of 3D Monte Carlo-based scatter correction for $99 \mathrm{mTc}$ cardiac perfusion SPECT. J Nucl Med 2006;47:1662-9.

8. Daou D, Pointurier I, Coaguila C, Vilain D, Benada AW, Lebtahi $\mathrm{R}$, et al. Performance of OSEM and depth-dependent resolution recovery algorithms for the evaluation of global left ventricular function in $201 \mathrm{Tl}$ gated myocardial perfusion SPECT. J Nucl Med 2003;44:155-62.

9. Garcia EV, Tsukerman L, Keidar Z. A new solid state, ultra fast cardiac multi-detector SPECT system [abstract]. J Nucl Cardiol 2008;15:S3.

10. Kennedy JA, Yosilevsky G, Przewloka K, Israel O, Frenkel A. 3D spatial resolution map and sensitivity characterization of a dedicated cardiac CZT SPECT camera [abstract]. J Nucl Med 2009;50:107P

11. Gambhir SS, Berman DS, Ziffer J, Nagler M, Sandler M, Patton J, et al. A novel high-sensitivity rapid-acquisition single-photon cardiac imaging camera. J Nucl Med 2009;50:635-43.

12. Kadrmas DJ, Frey EC, Tsui BM. Simultaneous technetium-99m/ thallium-201 SPECT imaging with model-based compensation for cross-contaminating effects. Phys Med Biol 1999;44:1843-60.

13. Dilsizian V, Rocco TP, Freedman NM, Leon MB, Bonow RO. Enhanced detection of ischemic but viable myocardium by the reinjection of thallium after stress-redistribution imaging. $\mathrm{N}$ Engl $\mathrm{J}$ Med 1990;323:141-6.

14. Johnson LL, Verdesca SA, Aude WY, Xavier RC, Nott LT, Campanella MW, et al. Postischemic stunning can affect left ventricular ejection fraction and regional wall motion on poststress gated sestamibi tomograms. J Am Coll Cardiol 1997;30:1641-8.

15. Seto H, Shimizu M, Nozawa T. Simultaneous assessment of regional adrenergic activity and perfusion with 123I-MIBG and 201Tl in congestive heart failure. Nucl Med Commun 1996; 17:225-30.

16. Borges-Neto S, Pagnanelli RA, Shaw LK, Honeycutt E, Shwartz SC, Adams GL, et al. Clinical results of a novel wide beam reconstruction method for shortening scan time of Tc-99m cardiac SPECT perfusion studies. J Nucl Cardiol 2007;14:555-65.

17. DePuey EG, Gadiraju R, Clark J, Thompson L, Anstett F, Shwartz SC. Ordered subset expectation maximization and wide beam reconstruction "half-time", gated myocardial perfusion SPECT functional imaging: A comparison to "full-time", filtered backprojection. J Nucl Cardiol 2008;15:547-63.

18. Ali I, Ruddy TD, Almgrahi A, Anstett FG, Wells RG. Half-time SPECT myocardial perfusion imaging with attenuation correction. J Nucl Med 2009;50:554-62.

19. Maddahi J, Mendez R, Mahmarian JJ, Thomas G, Babla H, Bai C, et al. Prospective multicenter evaluation of rapid, gated SPECT myocardial perfusion upright imaging. J Nucl Cardiol 2009;16:351-7.

20. Madias JE. Falsely negative thallium-201 scintigram associated with truly positive exercise electrocardiogram: The case of the globally balanced myocardial ischemia. Cardiology 2006;105:22-4.

21. Lesser JR, Bae R, Flygenring B, Sharkey SS, Lindberg J, Schwartz RS. Balanced myocardial ischaemia: A case of "normal" stress Tc99 sestamibi scan and diagnosis. Heart 2005;91:e53.

22. Kawai Y, Tsukamoto E, Nozaki Y, Morita K, Sakurai M, Tamaki N. Significance of reduced uptake of iodinated fatty acid analogue for the evaluation of patients with acute chest pain. J Am Coll Cardiol 2001;38:1888-94.

23. Ben-Haim S, Van Gramberg D, Bomanji J, Prvulovich E, Groves A, Kayani I, et al. Thallium 201 (Tl) myocardial perfusion imaging (MPI) with a novel fast cardiac camera versus conventional camera (SPECT) [abstract]. J Nucl Med 2009;50:124P.

24. Keidar Z, Kagna O, Frenkel A, Israel O. A novel ultrafast cardiac scanner for myocardial perfusion imaging (MPI): Comparison with a standard dual-head camera [abstract]. J Nucl Med 2009;50:125P.

25. Sharir T, Ben-Haim S, Merzon K, Prochorov V, Dickman D, BenHaim $S$, et al. High-speed myocardial perfusion imaging initial clinical comparison with conventional dual detector anger camera imaging. J Am Coll Cardiol Imaging 2008;1:156-63.

26. Berman DS, Kang X, Tamarappoo B, Wolak A, Hayes SW, Nakazato R, et al. Stress thallium-201/rest technetium-99m sequential dual isotope high-speed myocardial perfusion imaging. J Am Coll Cardiol Imaging 2009;2:273-82.

27. Takahashi N, Bommireddipalli S, DePuey EG. 24-frame gated myocardial perfusion SPECT (GSPECT) with ordered subset expectation maximum (OSEM) and resolution recovery [abstract]. J Nucl Med 2009;50:204P.

28. Gelfand MJ, Lemen LC. PET/CT and SPECT/CT dosimetry in children: The challenge to the pediatric imager. Semin Nucl Med 2007;37:391-8.

29. Einstein AJ, Henzlova MJ, Rajagopalan S. Estimating risk of cancer associated with radiation exposure from 64-slice computed tomography coronary angiography. JAMA 2007;298:317-23.

30. Nickoloff EL, Alderson PO. A comparative study of thoracic radiation doses from 64-slice cardiac $\mathrm{CT}$. $\mathrm{Br} \mathrm{J}$ Radiol 2007;80:537-44.

31. Case J, Bateman T, Cullom S. Obtaining optimum and consistent SPEW myocardial counts using an anterior planar view to 
determine SPECT acquisition times [abstract]. J Am Coll Cardiol 1998;31:82A.

32. Germano G, Nichols KJ, Cullom SJ, Faber TL, Cooke CD. Gated perfusion SPECT: Technical considerations. In: DePuey EG, Berman DS, Garcia EV, editors. Cardiac SPECT. 2nd ed. Philadelphia: Lippincott, Williams \& Wilkins; 2001. p. 103-15.

33. Da Silva AJ, Tang HR, Wong KH, Wu MC, Dae MW, Hasegawa $\mathrm{BH}$. Absolute quantification of regional myocardial uptake of 99mTc-sestamibi with SPECT: Experimental validation in a porcine model. J Nucl Med 2001;42:772-9.

34. Iida H, Eberl S, Kim KM, Tamura Y, Ono Y, Nakazawa M, et al. Absolute quantitation of myocardial blood flow with (201)Tl and dynamic SPECT in canine: Optimisation and validation of kinetic modeling. Eur J Nucl Med Mol Imaging 2008;35:896-905.

35. Herrero P, Markham J, Shelton ME, Weinheimer CJ, Bergmann SR. Noninvasive quantification of regional myocardial perfusion with rubidium- 82 and positron emission tomography exploration of a mathematical model. Circulation 1990;82:1377-86.

36. El Fakhri G, Sitek A, Guerin B, Kijewski MF, Di Carli MF, Moore SC. Quantitative dynamic cardiac 82Rb PET using generalized factor and compartment analyses. J Nucl Med 2005;46:1264-71.

37. Gould KL, Kirkeeide RL, Buchi M. Coronary flow reserve as a physiologic measure of stenosis severity. J Am Coll Cardiol 1990;15:459-74.

38. Demer LL, Gould KL, Goldstein RA, Kirkeeide RL, Mullani NA, Smalling RW, et al. Assessment of coronary artery disease severity by positron emission tomography. Comparison with quantitative arteriography in 193 patients. Circulation 1989; 79:825-35.
39. Hoffman EJ, Huang SC, Phelps ME. Quantitation in positron emission computed tomography: I. Effects of object size. J Comput Assist Tomogr 1979;5:391-400.

40. Nichols K, DePuey EG, Friedman MI, Rozanski A. Do patient data ever exceed the partial volume limit? J Nucl Cardiol 1998;5:48490.

41. Mariano-Goulart D, Dechaux L, Rouzet F, Barbotte E, Caderas de Kerleau C, Rossi M, et al. Diagnosis of diffuse and localized arrhythmogenic right ventricular dysplasia by gated blood-pool SPECT. J Nucl Med 2007;48:1416-23.

42. Nichols KJ, Van Tosh A, De Bondt P, Bergmann SR, Palestro CJ, Reichek N. Normal limits of gated blood pool SPECT count-based regional cardiac function parameters. Int $\mathrm{J}$ Cardiovasc Imaging 2008;24:717-25.

43. Galt JR, Garcia EV, Robbins WL. Effects of myocardial wall thickness on SPECT quantification. IEEE Trans Med Imaging 1990;9:144-50.

44. Germano G, Kiat H, Kavanagh PB, Mazzanti M, Su HT, Van Train KF, et al. Automatic quantification of ejection fraction from gated myocardial perfusion SPECT. J Nucl Med 1995;36:2138-47.

45. Faber TL, Cooke CD, Folks RD, Vansant JP, Nichols KJ, DePuey $\mathrm{EG}$, et al. Left ventricular function and perfusion from gated SPECT perfusion images: An integrated method. J Nucl Med 1999;40:650-9.

46. Friedman J, Van Train K, Maddahi J, Rozanski A, Prigent F, Bietendorf J, et al. "Upward creep" of the heart: A frequent source of false-positive reversible defects during thallium-201 stress-redistribution SPECT. J Nucl Med 1989;30:1718-22. 